\title{
Different orientations of sub-two-point threshold tactile stimuli can be discriminated
}

\author{
BARRY L. RICHARDSON and DIANNE B. WUILLEMIN \\ University of Papua New Guinea, Papua New Guinea
}

\begin{abstract}
The two points of an esthesiometer were touched on the axes of a cross drawn on the inside surface of subjects' forearms. One axis was drawn transversely (Line AB) and the other proximodistally (Line CD). Esthesiometer points (delivered simultaneously either on Line AB or CD) were always equidistant from the center of the cross, but they were reduced in separation distance until the two points felt like one (two-point threshold condition). A further condition consisted of the simultaneous delivery of two-point stimuli on Lines AB and CD in random order. Point separation was reduced until subjects could no longer report whether the two points touched were on Line $A B$ or Line $C D$ (orientation discrimination threshold). The latter threshold was found to be significantly lower than the two-point threshold, and this result was interpreted as support for the hypothesis that these two measures of tactile resolution take advantage of different properties of cutaneous receptive fields.
\end{abstract}

There are some grounds for doubting the adequacy of two-point threshold (2PT) measures as an index of tactile sensitivity. It has been pointed out that subjects may respond to the perceived size of a stimulus rather than its unity or duality (Boring, 1942), and it has been shown that training and set can effect a change in the measured 2PT values (Boring, 1942; Dresslar, 1894; Sen, 1966). But, more important, there are apparently more sensitive measures of tactile acuity. For example, the threshold for tactile size discrimination has been shown to be roughly 10 times smaller than the 2PT (Vierck \& Jones, 1969), and Loomis and Collins (1978) have demonstrated several instances of what they call tactile "hyperacuity." In one example, the threshold for detection of misalignment of three dots was found to be less than $10 \%$ of the $2 \mathrm{PT}$. Less dramatic but very reliable differences have also been found between the 2PT and the point localization threshold, or error of localization (Boring, 1942; Weinstein, 1968).

However, the task of interpreting observed differences between 2PT and discrimination measures of tactile acuity in terms of underlying physiological mechanisms is complicated by (among other things) the very difference between two points as a stimulus (for 2PT tests) and the myriad other stimuli that can be devised to impress upon the skin's surface for discrimination tests. Invariably, the to-be-discriminated stimuli are quantitatively and qualitatively different from two points and stimulate totally different regions of the integument (see Loomis \& Collins, 1978, for a discussion of various methods of testing tactile acuity). One consequence of this is some uncertainty about whether differences are attributable to a difference in measuring techniques or to different physiological factors involved in the two types of task. The present experiment was designed to essentially equate the stimuli used for determination of $2 \mathrm{PT}$ and discrimination threshold so that observed differences might be more readily interpreted.

\section{METHOD}

\section{Subjects}

The subjects were eight female and six male undergraduates attending Acadia University, Nova Scotia. Ages ranged from 18 to 27 years.

\section{Apparatus and Procedure}

A cross was drawn on the inside (volar) surface of the forearm of each subject. Each axis of the cross was $3 \mathrm{in.} \mathrm{long,} \mathrm{and}$ one was drawn transversely (labeled $\mathrm{AB}$ ) and the other proximodistally (CD). These lines intersected each other at their midpoints. Using a modified method of limits (see Murray, Ward, \& Hockley, 1975), the 2PT was established along the lines AB and CD, respectively. Subjects were instructed to say "two" only when two distinct points could be felt. In a descending series, point separation was reduced until the subject said "one" in response to two points, at which time the separation distance was increased and an ascending series began. This series continued until the subject said "two." The two points of stimulation were always equidistant from the center of the cross (i.e., the two points straddled the center of the cross), and each 2PT measure was calculated as the average of two ascending and two descending series with steps of $1 / 16 \mathrm{in}$. $(1.59 \mathrm{~mm})$.

Following measurement of each subject's 2PT (one for Line $A B$ and the other for Line $C D$ ), each subject was required to discriminate between the orientations of two two-point stimuli. For this purpose, two points, again equidistant from the center of the cross, were simultaneously touched on either Line $A B$ or Line $C D$, depending on the previously established random-order schedule (i.e., successively delivered $A B$ and $C D$ trials were interspersed according to a random schedule).

On the first trial, the esthesiometer was set with its two points at $1 / 8$ in. $(3.2 \mathrm{~mm})$ above the subject's already measured 2PT for that line. If the subject correctly reported the two points touched to be on, say, Line $A B$, the separation distance for the next trial on Line $A B$ was reduced by $1 / 8$ in. The same procedure was followed for stimuli on Line CD (i.e., the first two-point stimulus was set at $1 / 8$ in. above the $2 \mathrm{PT}$ already 
measured for that subject along Line CD). Reduction of twopoint separation continued with each successive trial until an error (e.g., saying " $A B$ " when in fact the line touched was $C D$ ) occurred, at which time, separation distance was increased, ready for the next trial on that particular line (either $\mathrm{AB}$ or $\mathrm{CD}$ ). This procedure was followed until two ascending and two descending series had been completed for both lines. Orientation discrimination thresholds for $\mathrm{AB}$ and $\mathrm{CD}$ (transverse and proximodistal) axes were independently calculated as the mean of the four respective measures (i.e., two ascending and two descending) thus obtained.

Finally, following the orientation discrimination condition, each subject's 2PT was again tested in the manner described earlier. The mean of these two 2PT measures (i.e., pre- and postdiscrimination trials) was used as the final measure of 2PT, against which orientation discrimination performance was then compared. Measures of 2PT were taken before and after the orientation discrimination trials in order to take in to account possible practice effects.

\section{RESULTS AND DISCUSSION}

The means of the pre- and postorientation discrimination measures of 2PT varied by less than $1 \mathrm{~mm}$, suggesting no practice effects.

The mean 2PTs for the $\mathrm{AB}$ and $\mathrm{CD}$ axes were $23.0 \mathrm{~mm}$ and $37.6 \mathrm{~mm}$, respectively; the mean orientation discrimination thresholds were $20.0 \mathrm{~mm}(\mathrm{AB})$ and $24.0 \mathrm{~mm}$ (CD). The overall mean orientation discrimination threshold was significantly lower than the overall mean $2 \mathrm{PT}$ $[t(13)=4.33, p<.001]$. This difference is represented in Figure 1, which shows (with smoothed curves) the effect of reducing the point separation distance on (1) the percentage of subjects capable of resolving two points and (2) the percentage of subjects capable of discriminating between the orientations of two twopoint stimuli.

An interesting result is that the orientation of two points can apparently be discriminated within a region of skin that, according to 2PT measures, should yield a

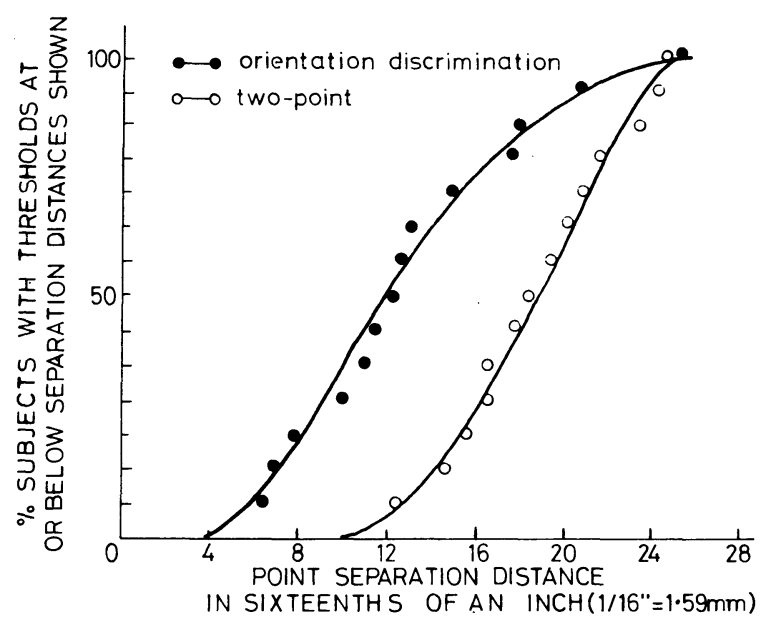

Figure 1. "Smoothed" curves showing the percentage of subjects capable of resolving two points (in the 2PT task) and percentage of subjects capable of discriminating between the orientations of two two-point stimuli, as a function of point separation distance. sensation of one point. The probable explanation for this finding is in accord with that suggested by other investigators, that is, that "twoness" requires the presence of a relatively silent receptive field between the two points of stimulation (Vallbo \& Johansson, 1978). On the other hand, orientation discrimination may merely require that different sets of receptive fields be involved when the to-be-discriminated pairs of stimuli are presented.

That is, discrimination measures of tactile sensitivity may take advantage of the position of overlapping receptive fields more than is the case with 2PT measures.

Only 1 of the 14 subjects showed a mean 2PT lower than the mean orientation discrimination threshold (difference of $.5 \mathrm{~mm}$ ).

The results are in agreement with previous studies in which it has been shown that discrimination threshold is lower than the 2PT. However, in the present study, the same region of skin was tested and two points of stimulation were used for both conditions. The means of the two 2PT measures observed along Lines $\mathrm{AB}$ and $\mathrm{CD}$ was $(23 \mathrm{~mm}+37.6 \mathrm{~mm}) / 2=30.3 \mathrm{~mm}$. This is in close agreement with other researchers' measures for the same skin region (e.g., Murray et al., 1975, reported a 2PT of $29.7 \mathrm{~mm}$ for the forearm). Also consistent with previous research was the finding that the longitudinal 2PT was greater than the transverse 2PT (Boring, 1921), and this supports a previously suggested relationship between 2PT and the characteristics of receptive fields that are typically oval in shape with the longer axis positioned proximodistally (Mountcastle, 1961; Sakata \& Iwamura, 1978). More specifically, discrimination tasks may involve judgments based upon different groups of receptive fields being active in the to-bediscriminated stimuli, whereas 2PT tasks involve decisions based on more restrictive criteria.

To clarify this idea, Figure 2 shows a hypothetical arrangement of receptive fields.

The precise shape and position of each receptive field is arbitrary, although some research supports the general configuration suggested here (Mountcastle, 1961; Sakata \& Iwamura, 1978). If a cross AB-CD is drawn on the skin and the receptive fields beneath it are as shown in Figure 2, certain predictions can be made, based on the suggestions above, about the size of the 2PT that should result from stimuli presented along the lines $A B$ and $C D$. For example, if we begin by touching two points on $A B$ such that they are equidistant from the center of the cross but outside Receptive Field 6, the two points should be felt as two, since they activate two spatially separate fields (5 and 3 ). However, as the points are moved closer to the center of the cross (still maintaining an equal distance between the center of the cross and the two points of stimulation), they will eventually both be within Field 6 , and there will therefore no longer be a relatively "silent" receptive field between them. At this point, Fields 5, 6, and 3 will be activated, but the "distinctiveness" or the "twoness" of the stimulus will have gone. 


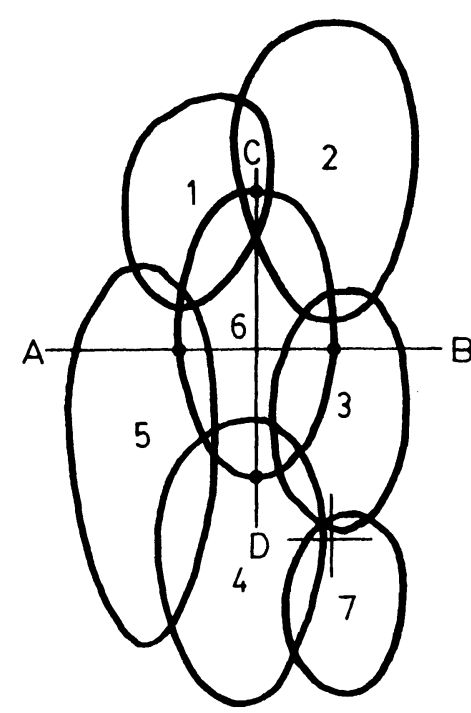

Figure 2. Hypothetical arrangement of overlapping receptive fields. The filled circles indicate the positions on the axes of the cross corresponding to the 2PT, which is dictated by the size of Receptive Field 6. (Although the cross center is "conveniently" shown to fall in the center of Field 6, the general principles outlined in the text should apply for any location on the cross. The particular position chosen was for the sake of descriptive convenience.)

Similar effects should occur for two-point stimuli presented on Line CD. That is, when the two points of stimulation are outside Field 6, one point will fall within Fields 1 and 2 and the other in Field 4, leaving Field 6 unstimulated. Thus, the size of the 2PT along Lines $\mathrm{AB}$ and $\mathrm{CD}$ will be determined by the size and position of the potentially silent receptive field between the two points of stimulation.

A very different prediction applies if the task is one of discriminating between the orientations of two twopoint stimuli (similar to those used to establish 2PT), one pair delivered on Line $\mathrm{AB}$ and the other on Line CD.

The threshold for this task should, according to the present hypothesis, be lower than the 2PT, for the following reason. As two-point stimuli on, say, $C D$, are systematically brought closer to the center of the cross, there will come a moment at which at least one of the two points is within Field 6 (this is the point at which "twoness" might be lost). However, at this stage, Fields 1, 2, 4, and 6 are active, and such activity within this ensemble of fields might be readily discriminable from activity within Fields 3, 5, and 6, which could result from two-point stimuli being applied on Line AB. That is to say, whereas the distinct impression of two points may be lost when no "silent" field exists between them, orientation discrimination should not fail until both pairs of stimuli are so far within Receptive Field 6 that no other field is getting any stimulation.

The results of the present experiment support this interpretation. However, the similarity of the stimuli used in the two experimental conditions implies that the performance differences observed were primarily a function of instructional set, with the same physiological information available in both cases, but differently used.

That is, since the same skin locations were touched in the two experimental conditions, it seems reasonable to suppose that the same information was available with each individual two-point stimulus but what the subject was required to do with that information varied. Relevant to this, Boring (1921) reported that when two points were so close as to be nondistinct perceptually, there was nevertheless an "ovality" or "elongation" of the stimulus before it really felt like one (at much smaller separation distances). Such an experience could be expected to result from the stimulation of two points that fall within two or more overlapping receptive fields with no "silent" field between them. (This idea is similar to that originally proposed by Weber; see Boring, 1942.)

Thus, it seems possible that the ovality or extension experienced when at least two adjacent fields were activated had a perceived orientation that served as a basis for discrimination in the present experiment (provided that different fields were stimulated when the two-point orientation was changed). Theoretically, the same ovality cues could have been used to discriminate between one and two points of stimulation (even though the impression of two distinct points was absent), and if they were, there should have been no difference between the 2PT and orientation discrimination threshold. The fact of the observed difference presumably reflected the subjects' attempts to comply with the instruction to say "two" only when two distinct points could be perceived and to ignore the "extended one" percept in the 2PT condition.

If this is correct, the extent of the difference between 2PT and orientation discrimination threshold should reflect the extent of receptive field overlap for the particular region of skin tested. Of course, this is an oversimplification, if only because there was no way of knowing where the center of the cross was drawn in relation to the receptive fields beneath it. However, the mean of the smallest point separations that were successfully discriminated with respect to their orientation was about half the smallest mean 2PT measured (see Figure 1). The suggestion here is that orientation discrimination threshold could, theoretically, be very low indeed if the center of the cross happened to be, for example, positioned as shown within Field 7 in Figure 2. In contrast, two-point resolution should be relatively unaffected.

In conclusion, this study has shown that discrimination between pairs of two-point stimuli is possible when the orientation of these stimuli is changed but delivered within an area of skin in which, according to 2PT measures, two points should feel like one point. Regardless of the presence or absence of an associated experience of ovality, it seems possible that orientation dis- 
crimination was based on the fact that different receptive fields were stimulated when the two-point orientation was changed. A similar principle, the recruitment of different receptive fields with small changes in the size or position of to-be-discriminated stimuli, may account for other instances in which discrimination acuity is better than two-point resolution.

\section{REFERENCES}

Boring, E. G. The stimulus error. American Journal of Psychology, 1921, 32, 449-471.

Boring, E. G. Sensation and perception in the history of experimental psychology. New York: Appleton-Century-Crofts, 1942.

Dresslar, F. B. Studies in the psychology of touch. American Journal of Psychology, 1894, 18, 313-368.

Loomis, J. M., \& Collins, C. C. Sensitivity to shifts of a point stimulus: An instance of tactile hyperacuity. Perception \& Psychophysics, 1978, 24, 487-492.
Murray, D., WARd, R., \& HockLey, W. Short term memory for tactile stimuli. Quarterly Journal of Experimental Psychology, 1975, 27, 303-312.

Mountcastue, V. B. Some functional properties of the somatic afferent system. In W. A. Rosenblith (Ed.), Sensory communication. Cambridge, Mass: M.I.T. Press, 1961.

SAKATA, H., \& I wamura, Y. Cortical processing of tactile information in the first somatosensory and parietal association areas in the monkey. In G. Gordon (Ed.), Active touch. Oxford: Pergamon Press, 1978.

SEN, A. The effects of set on the two-point threshold. Quarterly Journal of Experimental Psychology, 1966, 18, 169-174.

Vallbo, A. B., \& Johansson, R. S. Tactile sensory innervation of the glabrous skin of the human hand. In G. Gordon (Ed.), Active touch. Oxford: Pergamon Press, 1978.

Vierck, C. J., \& Jones, M. B. Size discrimination on the skin. Science, 1969, 63, 488-489.

WEINSTEIN, $\mathbf{S}$. Intensive and extensive aspects of tactile sensitivity as a function of body part, sex, and laterality. In D. R. Kenshalo (Ed.), The skin senses. Springfield, Ill: Thomas, 1968.

(Received for publication September 21, 1981.) 